\title{
Interfacial energies on quasicrystals
}

\author{
Andrea Braides \\ Dipartimento di Matematica, Università di Roma Tor Vergata \\ via della ricerca scientifica 1, 00133 Roma, Italy \\ Andrea Causin and Margherita Solci \\ DAP, Università di Sassari \\ piazza Duomo 6, 07041 Alghero (SS), Italy
}

\section{Introduction}

There are several ways to mathematically define quasicrystalline patterns. We adopt here the "cut and project" approach, where a quasicrystal consists of the projection of a 'stripe' of a higher-dimensional periodic pattern. If the direction of this stripe is irrational with respect to the periods of the original pattern then the resulting projected structure is not periodic, lacking translational symmetry. However, it inherits some 'quasiperiodic' properties from the original lattice. In the case of a Penrose lattice, for example, we have a five-fold symmetry deriving from its description as the projection from a five-dimensional lattice on a twodimensional plane.

Our notation for the "cut and project" approach is as follows. We fix an $n$-dimensional plane $\Pi_{n} \subset \mathbb{R}^{N}$, and suppose that it is an "irrational" plane; i.e., that $\Pi_{n} \cap \mathbb{Z}^{N}=\{0\}$. This is not a restriction of our method: in the case that $\Pi_{n} \cap \mathbb{Z}^{N}$ is a $n$-dimensional lattice then we are in a simpler periodic setting, while the case when $\Pi_{n} \cap \mathbb{Z}^{N}$ is a $k$-dimensional lattice with $0<k<n$ can be seen as a combination of the extreme cases. We then consider a fixed polyhedral neighbourhood of $\Pi_{n}, S=\Pi_{n} \oplus S_{0}$ with $S_{0}$ a polyhedral set in the orthogonal complement of $\Pi_{n}$, and $\Sigma=\mathbb{Z}^{N} \cap S$. A pictorical description for $N=3$ and $n=1$ is given in Fig. 1.

We will consider interactions corresponding to nearest neighbours in $\Sigma$ (i.e., pairs $(i, j)$ with $|i-j|=1)$. In order that this system of interactions give rise to a quasicrystal we have to assume that $\Sigma$ be large enough so that we do not have "disconnected" points inside $\Sigma$. To that end, we assume that there exists a coordinate $n$-dimensional plane in $\mathbb{R}^{N}$, which we identify with $\mathbb{R}^{n}$ such that the projection $P: \mathbb{Z}^{N} \rightarrow \mathbb{Z}^{n} \subset \mathbb{R}^{n}$ satisfies:

- for all nearest neighbours $\alpha, \beta \in \mathbb{Z}^{n}$ there exists at least a pair of nearest neighbours $i_{\alpha} \in P^{-1}(\alpha) \cap \Sigma$ and $i_{\beta} \in P^{-1}(\beta) \cap \Sigma$.

We will consider a "ferromagnetic" spin energy defined on $\Sigma$ of the form

$$
\sum_{i, j}\left(u_{i}-u_{j}\right)^{2}, \quad u: \Sigma \rightarrow\{-1,1\}
$$




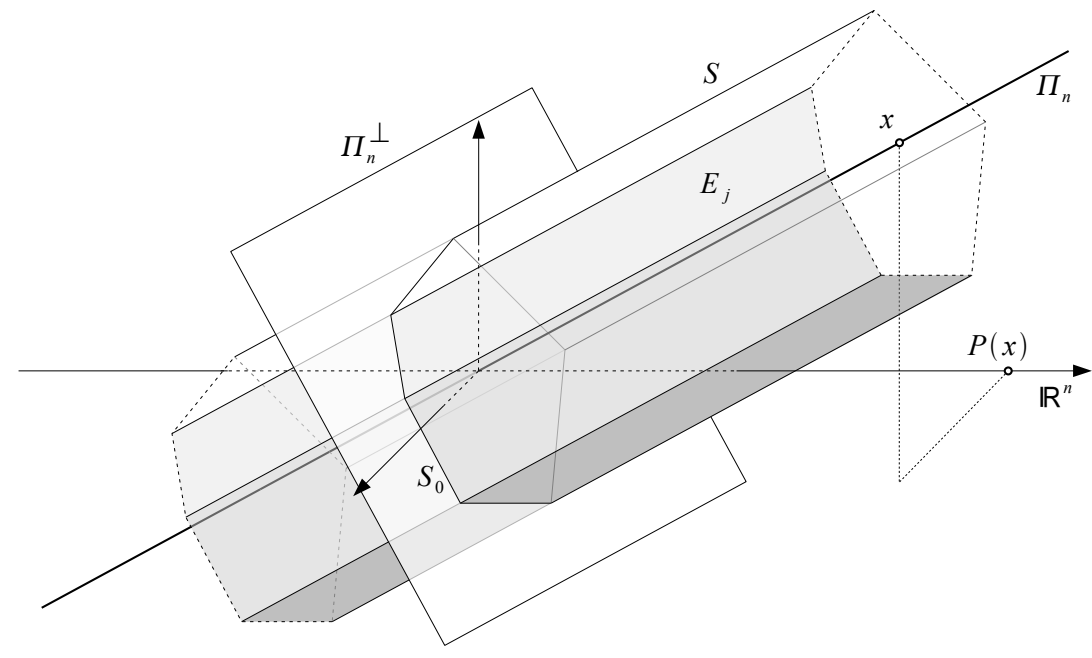

Figure 1: Picture of $S(n=1, N=3)$

where the sum runs on nearest neighbours in $\Sigma$ (note that on bounded sets this energy can be rewritten in the more common form $-2 \sum_{i, j} u_{i} u_{j}$, up to an additive constant. We prefer (1) so that uniform states have zero energy), and describe its "macroscopic" behaviour. To this end, we introduce a small parameter $\varepsilon>0$ and scale the energies as

$$
F_{\varepsilon}(u)=\sum_{i, j} \varepsilon^{n-1}\left(u_{i}-u_{j}\right)^{2}, \quad u: \varepsilon \Sigma \rightarrow\{-1,1\} .
$$

The scaling is justified by recalling that in the periodic setting with $n=N$ this normalization gives a non-trivial surface energy in the limit (see [1]). Note that our problem can be interpreted as the asymptotic description of a "discrete thin film" concentrating on the plane $\Pi_{n}$ (see e.g. [7]).

We will prove that, as $\varepsilon \rightarrow 0$, energies (2) can be approximated by an interfacial energy. First, we prove a coerciveness property showing that sequences $\left\{u_{\varepsilon}\right\}$ equibounded in energy can be identified with sequences $\left\{v_{\varepsilon}\right\}$ defined on $\mathbb{R}^{n}$ converging to a limit $v: \mathbb{R}^{n} \rightarrow\{ \pm 1\}$ (or, equivalently $v: \Pi_{n} \rightarrow\{ \pm 1\}$ ). Every such $v$ is then identified with the set $A=\{v=1\}$; the energies $F_{\varepsilon}$ are then shown to converge to an interfacial integral of the form

$$
F(A)=\int_{\partial^{*} A} \varphi(\nu) d \mathcal{H}^{n-1} .
$$

A key point is the description of the surface tension $\varphi$, which is characterized by an asymptotic formula involving discrete optimal transition problems on large sets in $\Sigma$, corresponding to large cubes in $\mathbb{R}^{n}$, similar to those introduced in [7] to deal with homogenization problems on continuous thin films. The main technical issue of the paper is precisely the proof of the existence of such $\varphi$, for which we adapt the quasiperiodic arguments of [11]. 
To this end we make a technical hypothesis by requiring that the boundary of the stripe $S$ be itself composed of portions of irrational hyperplanes. Under this assumption we prove a coarse-grained "almost invariance" of the energies for a wide set of translations; this is a consequence of the fact that for all $\eta>0$ we may find a uniformly dense set of "almost periods" $\sigma_{\eta}$ in $\mathbb{Z}^{N}$ such that translations of the stripe $\Sigma$ by $\sigma_{\eta}$ superposes exactly with $\Sigma$ except for a set of points whose location can be controlled. As a consequence, the effect on the translations of minimum problems can be estimated, which is a key point in the proof of asymptotic homogenization formulas.

We note that, contrary to the case of Penrose lattices considered in [11] where one can essentially reason directly on the projection of the higher-dimensional lattice on $\Pi_{n}$, here the description of the system as a discrete thin film is exploited both in the proof of the coerciveness of the energies and in the control over translations.

\section{Notation and setting of the problem}

Let $N>n \geq 1$; we fix $\Pi_{n}$ an $n$-dimensional linear subspace in $\mathbb{R}^{N}$. As already remarked in the introduction the relevant case is when

$$
\Pi_{n} \cap \mathbb{Z}^{N}=\{0\} .
$$

Otherwise, there exists $0<k \leq n$ such that we can write $\Pi_{n}=\Pi_{k} \oplus \Pi_{n-k}$, where $\Pi_{k}$ is a $k$-dimensional rational space; i.e., generated by $k$ independent vectors of $\mathbb{Z}^{N}$, and $\Pi_{n-k}$ is an $n-k$-dimensional space with $\Pi_{n-k} \cap \mathbb{Z}^{N}=\{0\}$, and the analysis can be decoupled in the two spaces.

Given $\xi \neq 0$ in the orthogonal complement of $\Pi_{n}$, denoted by $\Pi_{n}^{\perp}$, let $H(\xi)$ be the half-space defined by

$$
H(\xi)=\left\{x \in \mathbb{R}^{N}:\langle x-\xi, \xi\rangle \leq 0\right\} .
$$

Note that this is a way of describing any closed half-space $\mathcal{H}$ containing 0 in its interior. It suffices to take the vector of minimal length in $\partial \mathcal{H}$ as $\xi$.

For $K \in \mathbb{N}$ we choose $\xi_{1}, \ldots, \xi_{K} \in \Pi_{n}^{\perp} \backslash\{0\}$ such that $\bigcap_{j=1}^{K} H\left(\xi_{j}\right) \subset\left\{x \in \mathbb{R}^{N}\right.$ : $\left.\operatorname{dist}\left(x, \Pi_{n}\right) \leq R\right\}$ for some $R>0$, and define

$$
S=\bigcap_{j=1}^{K} H\left(\xi_{j}\right), \quad \Sigma=\mathbb{Z}^{N} \cap S .
$$

Note that $S$ is a polyhedral neighbourhood of $\Pi_{n}$ with boundary faces

$$
E_{j}=S \cap\left(\left\{\left\langle x, \xi_{j}\right\rangle=0\right\}+\xi_{j}\right) .
$$

With these assumptions, we have that $S_{0}=S \cap \Pi_{n}^{\perp}$ is a compact convex polyhedral set. In Fig. 1 we picture $S$ for $N=3$ and $n=1$.

With a slight abuse of notation, we identify $\mathbb{R}^{n}$ with a coordinate $n$-dimensional space in $\mathbb{R}^{N}$, which we may suppose to be the one generated by the first $n$ coordinate basis vectors, and denote by $P$ the orthogonal projection on $\mathbb{R}^{n}$ from $\mathbb{R}^{N}$; i.e.,

$$
P\left(x_{1}, \ldots, x_{N}\right)=\left(x_{1}, \ldots, x_{n}\right) .
$$


Our main assumption is that $\Sigma$ is connected, in the sense that each pair of points in $\Sigma$ can be joined by a chain of nearest neighbours in $\Sigma$. This is assured by the following assumption on $P$ :

(H) for all nearest neighbours $\alpha, \beta \in \mathbb{Z}^{n}$ there exists at least a pair of nearest neighbours $i_{\alpha} \in P^{-1}(\alpha) \cap \Sigma$ and $i_{\beta} \in P^{-1}(\beta) \cap \Sigma$.

In addition, we choose $\xi_{j}$ such that

$$
\left\{x:\left\langle x, \xi_{j}\right\rangle=0\right\} \cap \mathbb{Z}^{N}=\{0\} \text { for all } j=1, \ldots, K .
$$

With this technical assumption all boundary faces $E_{j}$ of $S$ lie on irrational hyperplanes parallel to $\Pi_{n}$, that is affine hyperplanes parallel to irrational $N$-1-dimensional subspaces containing $\Pi_{n}$. This property will be used to ensure that translations along $\Pi_{n}$ do not "change much" the geometry of $\Sigma$.

We introduce a small parameter $\varepsilon>0$ and denote by $\mathcal{A}_{\varepsilon}$ the set of all functions $u: \varepsilon \Sigma \rightarrow$ $\{-1,+1\}$. If $\varepsilon=1$ we will write $\mathcal{A}_{1}=\mathcal{A}$. We define the energies $F_{\varepsilon}: \mathcal{A}_{\varepsilon} \rightarrow[0,+\infty]$ by

$$
F_{\varepsilon}(u)=\sum_{i, j} \varepsilon^{n-1}\left(u_{i}-u_{j}\right)^{2},
$$

where $u_{i}=u(\varepsilon i)$, and the sum runs on all pairs of nearest neighbours in $\Sigma$; i.e., $i, j \in \Sigma$ such that $|i-j|=1$. Note that if $F_{\varepsilon}(u)<+\infty$ then $u_{i}$ is constant outside a bounded subset of $\Sigma$.

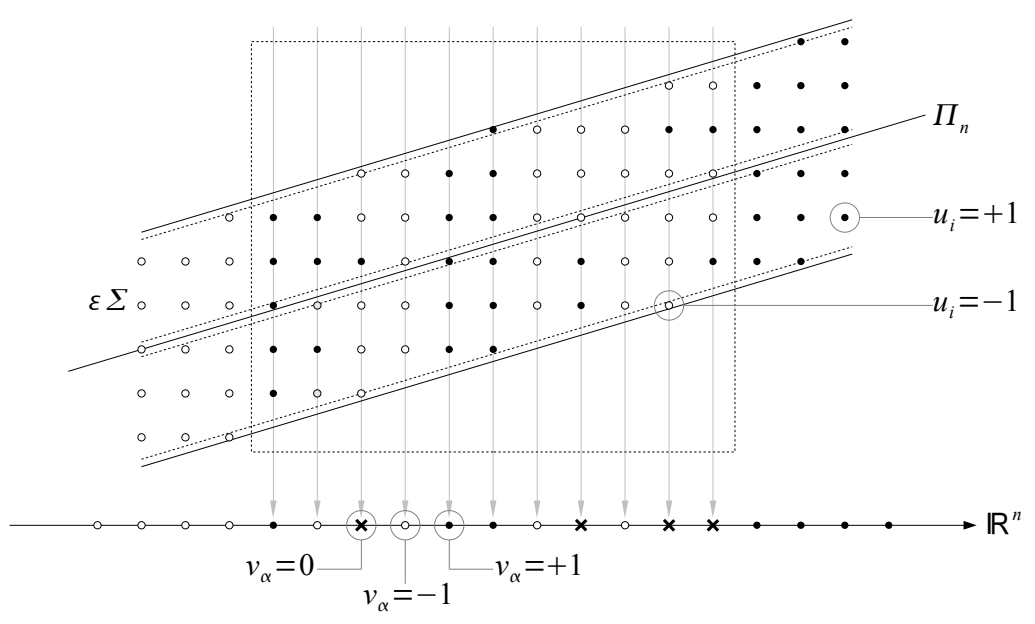

Figure 2: Projection from $\varepsilon \Sigma$ to $\varepsilon \mathbb{Z}^{n}(n=1, N=2)$

To each $u \in \mathcal{A}_{\varepsilon}$ we can associate a function $v=P(u): \varepsilon \mathbb{Z}^{n} \rightarrow\{-1,0,1\}$ as follows (see 
Fig. 2):

$$
v_{\alpha}=v(\varepsilon \alpha)= \begin{cases}1 & \text { if } u_{i}=u(\varepsilon i)=1 \text { for all } i \in P^{-1}(\alpha) \cap \Sigma \\ -1 & \text { if } u_{i}=u(\varepsilon i)=-1 \text { for all } i \in P^{-1}(\alpha) \cap \Sigma \\ 0 & \text { otherwise }\end{cases}
$$

In its turn each such $v$ is extended to a piecewise-constant function on $\mathbb{R}^{n}$; e.g., by

$$
v(x)=v_{\alpha} \text { if } x \in \varepsilon \alpha+\left(-\frac{\varepsilon}{2}, \frac{\varepsilon}{2}\right)^{n} .
$$

We have the following coerciveness result.

Proposition 1 (coerciveness). Let hypothesis $(\mathrm{H})$ hold. Let $\left\{u_{\varepsilon}\right\}$ be a sequence in $\mathcal{A}_{\varepsilon}$ with $\sup _{\varepsilon} F_{\varepsilon}\left(u_{\varepsilon}\right)<+\infty$, and let $v_{\varepsilon}=P\left(u_{\varepsilon}\right)$ be the corresponding piecewise-constant functions on $\mathbb{R}^{n}$ defined as in (9). Then the sequence $\left\{v_{\varepsilon}\right\}$ is strongly precompact in $L_{\text {loc }}^{1}\left(\mathbb{R}^{n}\right)$, and each its limit belongs to $B V\left(\mathbb{R}^{n} ;\{-1,1\}\right)$.

Proof. Proposition 1 follows easily by estimating the perimeter of the sets $\left\{v_{\varepsilon}= \pm 1\right\}$ and showing that the sets $\left\{v_{\varepsilon}=0\right\}$ are asymptotically negligible.

We will use the notation $v_{\alpha}^{\varepsilon}:=\left(v_{\varepsilon}\right)_{\alpha}$ and $u_{i}^{\varepsilon}:=\left(u_{\varepsilon}\right)_{i}$. The letter $c$ will denote a strictly positive constant, whose value may change from line to line.

First, note that for all $\alpha \in \mathbb{Z}^{n}$ such that $v_{\alpha}^{\varepsilon}=0$ there exist nearest neighbours $i, j \in$ $P^{-1}(\alpha) \cap \Sigma$ such that $u_{i}^{\varepsilon} \neq u_{j}^{\varepsilon}$; hence

$$
4 \varepsilon^{n-1} \#\left\{\alpha: v_{\alpha}^{\varepsilon}=0\right\} \leq F_{\varepsilon}\left(u_{\varepsilon}\right) \leq c
$$

so that

$$
\varepsilon^{n-1} \#\left\{\alpha: v_{\alpha}^{\varepsilon}=0\right\} \leq c
$$

and

$$
\left|\left\{x \in \mathbb{R}^{n}:\left|v_{\varepsilon}(x)\right| \neq 1\right\}\right|=\left|\left\{x \in \mathbb{R}^{n}: v_{\varepsilon}(x)=0\right\}\right|=\varepsilon^{n} \#\left\{\alpha: v_{\alpha}^{\varepsilon}=0\right\} \leq c \varepsilon .
$$

It is sufficient then to prove that the (characteristic function of the) sets

$$
V_{\varepsilon}=\left\{x \in \mathbb{R}^{n}: v_{\varepsilon}(x)=1\right\}
$$

are pre-compact in $L_{\mathrm{loc}}^{1}\left(\mathbb{R}^{n}\right)$. To that end (see e.g. [3]) it suffices to prove that

$$
\mathcal{H}^{n-1}\left(\partial^{*} V_{\varepsilon}\right) \leq c .
$$

This follows immediately from the estimate

$$
\begin{aligned}
\mathcal{H}^{n-1}\left(\partial^{*} V_{\varepsilon}\right)= & \varepsilon^{n-1} \#\left\{(\alpha, \beta):|\alpha-\beta|=1, \alpha \in V_{\varepsilon}, \beta \notin V_{\varepsilon}\right\} \\
= & \varepsilon^{n-1}\left(\#\left\{(\alpha, \beta):|\alpha-\beta|=1, v_{\alpha}^{\varepsilon}=1, v_{\beta}^{\varepsilon}=0\right\}\right. \\
& \left.\quad+\#\left\{(\alpha, \beta):|\alpha-\beta|=1, v_{\alpha}^{\varepsilon}=1, v_{\beta}^{\varepsilon}=-1\right\}\right) \\
\leq & \varepsilon^{n-1}\left(\#\left\{\alpha: v_{\alpha}^{\varepsilon}=0\right\}\right. \\
& \left.\quad+\#\left\{(i, j):|i-j|=1, u_{i}^{\varepsilon}=1, u_{j}^{\varepsilon}=-1\right\}\right) \\
= & \varepsilon^{n-1} \#\left\{\alpha: v_{\alpha}^{\varepsilon}=0\right\}+\frac{1}{8} F_{\varepsilon}\left(u_{\varepsilon}\right) \leq c
\end{aligned}
$$


by $(10)$.

The coerciveness result in Proposition 1 justifies the definition of the convergence

$$
u_{\varepsilon} \rightarrow u \quad \Longleftrightarrow \quad P\left(u_{\varepsilon}\right) \rightarrow u \text { strongly in } L_{\text {loc }}^{1}\left(\mathbb{R}^{n}\right),
$$

where $u \in B V\left(\mathbb{R}^{n} ;\{-1,1\}\right)$, or, also, equivalently

$$
u_{\varepsilon} \rightarrow A \quad \Longleftrightarrow \quad u_{\varepsilon} \rightarrow u \text { and } u=-1+2 \chi_{A},
$$

where $A$ is a set of finite perimeter in $\mathbb{R}^{n}$.

Remark 2. The convergence above can also be interpreted as a 'thin-film convergence'; i.e. as a $L^{1}$-convergence on a fixed set obtained by scaling to a fixed size the components of points in $\varepsilon \Sigma$ orthogonal to $\Pi_{n}$ (see [7]; see also [4] Section 14). In our case the convergence may be considered in the set

$$
S_{\varrho}=\left\{x \in \mathbb{R}^{N}: \operatorname{dist}\left(x, \Pi_{n}\right)<\varrho\right\},
$$

where $\varrho$ is small enough so that $S_{\varrho}$ is contained in the union of all cubes $i+(-1 / 2,1 / 2)^{N}$ with $i \in \Sigma$ (if no such $\varrho$ exists then the definition of convergence is slightly more complex). We extend the functions $u \in \mathcal{A}_{\varepsilon}$ to piecewise-constant functions on the cubes $i+(-\varepsilon / 2, \varepsilon / 2)^{N}$. Note that $S_{\varrho}=S_{\varrho}^{\prime} \oplus \Pi_{n}$, where $S_{\varrho}^{\prime}$ is in the orthogonal complement of $\Pi_{n}$. If $x=y+s$ with $s \in S_{\varrho}^{\prime}$ and $y \in \Pi_{n}$, we set $\tilde{u}_{\varepsilon}(x)=u_{\varepsilon}(y+\varepsilon s)$. Then for sequences with equi-bounded energy we have $u_{\varepsilon} \rightarrow u$ if and only if $\tilde{u}_{\varepsilon} \rightarrow \tilde{u}$ on bounded subsets of $S_{\varrho}$, where $\tilde{u}(x)=u(P(y))$ depends only on $y \in \Pi_{n}$ if $x=y+s$.

We will describe the asymptotic behaviour of the energies $F_{\varepsilon}$ through the computation of their $\Gamma$-limit with respect to the convergence defined in (12). This limit will turn out to be a surface integral. In order to define the limit surface tension we introduce some notation: for fixed $\nu \in S^{n-1}$ we will denote by $Q^{\nu} \subset \mathbb{R}^{n}$ an $n$-cube centered in 0 with side length 1 and one face orthogonal to $\nu$. We also consider the function

$$
v^{\nu}(x)= \begin{cases}1 & \text { if }\langle x, \nu\rangle \geq 0 \\ -1 & \text { otherwise }\end{cases}
$$

If $E \subset \mathbb{Z}^{n}$ we will set

$$
\bar{E}=\left\{i \in \mathbb{Z}^{n}: \exists j \in E:|i-j| \leq 1\right\}
$$

and (with abuse of notation) for $E \subset \mathbb{R}^{n}$ we set $\bar{E}=\overline{E \cap \mathbb{Z}^{n}}$. Finally, we localize our energies on (pre-images of) subsets of $\mathbb{R}^{n}$ by setting

$$
F_{\varepsilon}(u, E)=\sum_{i, j \in \Sigma \cap P^{-1}(E)} \varepsilon^{n-1}\left(u_{i}-u_{j}\right)^{2},
$$

where the sum is taken on all nearest neighbours in $\Sigma \cap P^{-1}(E)$. If $\varepsilon=1$ we will write $F_{1}=F$. tion 4 .

With this notation we can state the following proposition, which will be proved in Sec- 
Proposition 3 (existence of the surface tension). For all $\nu \in S^{n-1}$ there exists the limit

$$
\lim _{T \rightarrow+\infty} \frac{1}{T^{n-1}} g_{T}(\nu)
$$

where

$$
g_{T}(\nu)=\min \left\{F\left(u, \overline{T Q^{\nu}}\right): u \in \mathcal{A}, P(u)=v^{\nu} \text { on } \mathbb{Z}^{n} \backslash T Q^{\nu}\right\} .
$$

The previous proposition allows us finally to state the main result of the paper.

Theorem 4 (homogenization of quasicrystalline lattices). Let $\Pi_{n}$ be a linear subspace of $\mathbb{R}^{N}$ of dimension $n$ satisfying $(4)$, let $\Sigma$ be defined by (5) and satisfy hypotheses $(\mathrm{H})$ and $(7)$. Let the energy $F_{\varepsilon}$ be defined by (8) and the surface tension $\varphi$ be defined by (15). Then the $\Gamma$-limit of $F_{\varepsilon}$ with respect to the convergence (12) is given by

$$
F_{0}(A)=\int_{\partial^{*} A} \varphi\left(\nu_{A}\right) d \mathcal{H}^{n-1},
$$

where $\partial^{*} A$ denotes the reduced boundary of $A$ and $\nu_{A}$ its interior normal.

The proof of this result will be obtained in Section 5 .

Remark 5. In the case $n=N-1$ hypothesis (7) is always satisfied thanks to (4) since the two faces of the boundary of $S$ are parallel to $\Pi_{n}$.

If we remove hypothesis (4) then $\Pi_{n}=\Pi_{k} \oplus \Pi_{n-k}$, where $\Pi_{k}$ is an irrational plane and $\Pi_{n-k}$ is a rational plane (i.e., $\Pi_{n-k} \cap \mathbb{Z}^{N}$ is a $n-k$-dimensional lattice). Then the same theorem holds provided that $S$ can be written as $\Pi_{n-k} \oplus S^{\prime}$, where $S^{\prime}$ satisfies (7) in the $N-n+k$ dimensional space orthogonal to $\Pi_{n-k}$. We will not deal with this case in detail.

It is not clear if (7) is just a technical hypothesis, and if the proof of the theorem can be adapted to cover non-polyhedral neighbourhoods of $\Pi_{n}$.

Remark 6 (local version of the homogenization theorem). In the same way as for Theorem 4 we can prove that for any fixed bounded open subset $\Omega$ in $\mathbb{R}^{n}$ with Lipschitz boundary we have convergence of the localized functionals $F_{\varepsilon}(\cdot, \Omega)$ to the localized interfacial energy

$$
F_{0}(A, \Omega)=\int_{\Omega \cap \partial^{*} A} \varphi\left(\nu_{A}\right) d \mathcal{H}^{n-1} .
$$

This can be proved by extending all functions to 1 outside $\varepsilon \Sigma \cap P^{-1}(\Omega)$ and using the theorem on compactly contained subsets of $\Omega$ to get a lower bound, while an upper bound is obtained by the same direct construction of the upper bound of the theorem. Note that in this case the $\Gamma$-limit is computed with respect to the convergence in $L^{1}(\Omega)$ in (11). 


\section{Quasiperiodic properties of quasicrystals}

In this section we derive some properties of $\Sigma$ when the plane $\Pi_{n}$ satisfies (4).

Proposition 7. For all $\eta>0$ the set

$$
T_{\eta}^{n}=\left\{\tau \in \mathbb{Z}^{n}: \operatorname{dist}\left(P^{-1}(\tau) \cap \Sigma, \Pi_{n}\right)<\eta\right\}
$$

is relatively dense in $\mathbb{R}^{n}$; i.e., there exists an inclusion length $L_{\eta}>0$ such that for all $x \in \mathbb{R}^{n}$ there exists $\tau \in T_{\eta}^{n}$ such that $x \in \tau+\left[0, L_{\eta}\right]^{n}$.

Proof. The function $f(x)=\operatorname{dist}\left(P^{-1}(x) \cap \Pi_{n}, \mathbb{Z}^{N}\right)$ is continuous and quasiperiodic; hence, it is uniformly almost-periodic in $\mathbb{R}^{n}$. By the characterization of uniformly almost-periodic functions in [2], the set $\widetilde{T}_{\eta}^{n}=\{x: f(x)<\eta\}$ is relatively dense in $\mathbb{R}^{n}$. Then $P\left(\left\{\sigma \in \mathbb{Z}^{N}\right.\right.$ : $\left.\left.\operatorname{dist}\left(\sigma, P^{-1}\left(\widetilde{T}_{\eta}^{n}\right) \cap \Pi_{n}\right)<\eta\right\}\right)$ is also relatively dense. Since by construction we have that

$$
\left\{\sigma \in \mathbb{Z}^{N}: \operatorname{dist}\left(\sigma, P^{-1}\left(\widetilde{T}_{\eta}^{n}\right) \cap \Pi_{n}\right)<\eta\right\}=\left\{\sigma \in \Sigma: \operatorname{dist}\left(\sigma, \Pi_{n}\right)<\eta\right\}
$$

then $T_{\eta}^{n}=P\left(\left\{\sigma \in \mathbb{Z}^{N}: \operatorname{dist}\left(\sigma, P^{-1}\left(\widetilde{T}_{\eta}^{n}\right) \cap \Pi_{n}\right)<\eta\right\}\right)$ concluding the proof.

We define

$$
T_{\eta}^{N}=\left\{\sigma \in \Sigma: \operatorname{dist}\left(\sigma, \Pi_{n}\right)<\eta\right\}
$$

so that $T_{\eta}^{n}=P\left(T_{\eta}^{N}\right)$. For any $\sigma \in T_{\eta}^{N}$ we define

$$
B(\sigma)=\left\{\alpha \in \mathbb{Z}^{n}:\left(P^{-1}(\alpha) \cap \Sigma\right)+\sigma \neq P^{-1}(\alpha+P(\sigma)) \cap \Sigma\right\}
$$

and

$$
B_{\eta}=\bigcup_{\sigma \in T_{\eta}^{N}} B(\sigma) .
$$

Proposition 8. There exist a sequence $\left\{R_{\eta}\right\}$ with $R_{\eta} \rightarrow+\infty$ as $\eta \rightarrow 0$, such that for any $x \in \mathbb{R}^{n}$ and any $\nu \in S^{n-1}$

$$
\#\left(B_{\eta} \cap\left(R_{\eta} Q^{\nu}+x\right)\right) \leq K
$$

where $K$ is the number of the faces of the neighbourhood $S$ of $\Pi_{n}$.

For $\eta>0$ and $j=1, \ldots, k$ we define

$$
\begin{aligned}
& \Lambda_{\eta}^{j}=\left\{i \in \mathbb{Z}^{N}: \operatorname{dist}\left(i, L_{j}+\xi_{j}\right)<\eta\right\} \quad \text { and } \quad \Lambda_{\eta}=\bigcup_{j=1}^{K} \Lambda_{\eta}^{j} \\
& H_{\eta}^{j}=\left\{x \in \mathbb{R}^{N}:\left\langle x-\xi_{j}, \xi_{j}\right\rangle<\eta\left|\xi_{j}\right|\right\} \text { and } S_{\eta}=\bigcap_{j=1}^{K} H_{\eta}^{j}
\end{aligned}
$$

where $L_{j}=\left\{x \in \mathbb{R}^{N}:\left\langle x, \xi_{j}\right\rangle=0\right\}$ (see Fig. 3). Note that $S_{\eta}$ is a polyhedral " $\eta$ neighbourhood" of $S$, so that $\Lambda_{\eta}$ are the points in $\mathbb{Z}^{N}$ lying in a polyhedral " $\eta$-neighbourhood" of $\partial S$. 


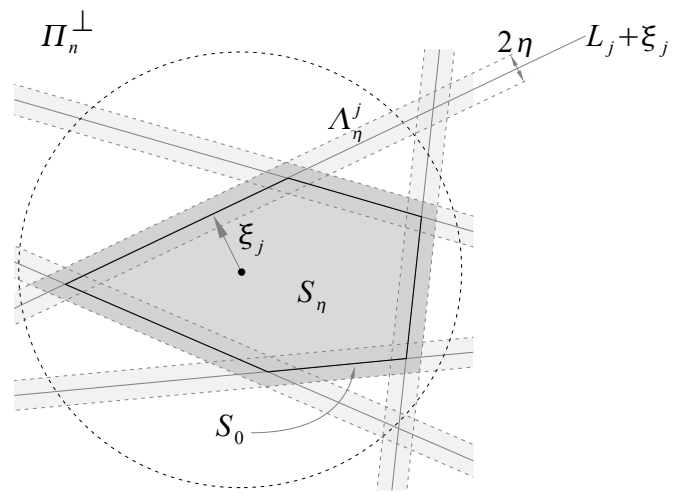

Figure 3: Parameterization of an $\eta$-neighbourhood of $S$

Proof. We start by showing that for any $\eta>0$

$$
B_{\eta} \subset P\left(\Lambda_{\eta} \cap S_{\eta}\right)
$$

(see Fig. 4). Indeed, suppose by contradiction that there exists $\alpha \in B_{\eta}$ such that for any

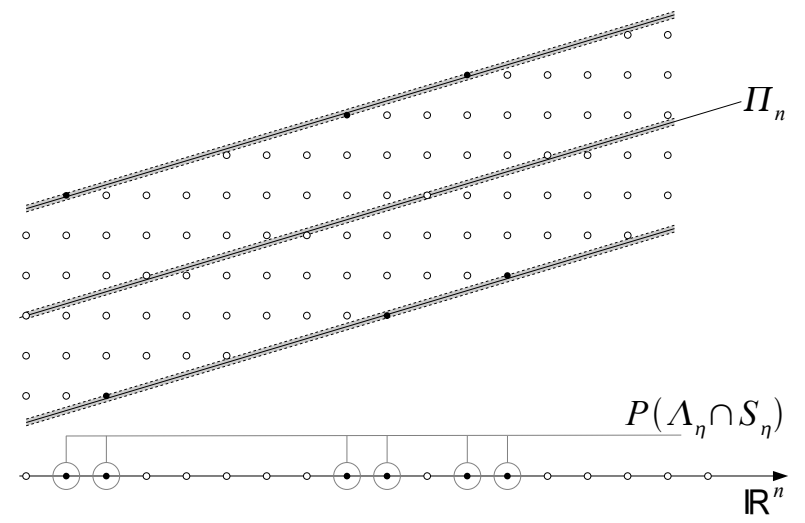

Figure 4: The set $P\left(\Lambda_{\eta} \cap S_{\eta}\right)$

$i \in P^{-1}(\alpha)$ either the inequality

$$
\operatorname{dist}\left(i, L_{j}+\xi_{j}\right)=\frac{\left|\left\langle i-\xi_{j}, \xi_{j}\right\rangle\right|}{\left|\xi_{j}\right|} \geq \eta \quad \text { holds for every } j=1, \ldots, K,
$$

or the inequality

$$
\left\langle i-\xi_{j}, \xi_{j}\right\rangle \geq \eta\left|\xi_{j}\right| \quad \text { holds for some } j \in\{1, \ldots, K\}
$$


Let $\sigma \in T_{\eta}^{N}$ be such that $\alpha \in B(\sigma)$. Then for any $j$ such that $\left|\left\langle i-\xi_{j}, \xi_{j}\right\rangle\right| \geq \eta\left|\xi_{j}\right|$, it follows that for $i \in P^{-1}(\alpha)$

$$
\eta^{2}\left|\xi_{j}\right|^{2}>\left|\left\langle\sigma, \xi_{j}\right\rangle\right|^{2} \geq \eta^{2}\left|\xi_{j}\right|^{2}-2\left\langle i+\sigma-\xi_{j}, \xi_{j}\right\rangle\left\langle i-\xi_{j}, \xi_{j}\right\rangle .
$$

This implies that $i$ and $i+\sigma$ belong to the same half-space $H\left(\xi_{j}\right)$ defined by $L_{j}+\xi_{j}$. If (22) holds, then (24) holds for any $j$, and we have

$$
i \in \Sigma \Longleftrightarrow i \in H\left(\xi_{j}\right) \text { for any } j \Longleftrightarrow i+\sigma \in H\left(\xi_{j}\right) \text { for any } j \Longleftrightarrow i+\sigma \in \Sigma \text {. }
$$

On the other hand, if (23) holds, then there exists an index $j$ such that (24) holds and $i \notin H\left(\xi_{j}\right)$. Thus, $i+\sigma \notin H\left(\xi_{j}\right)$, hence $i+\sigma \notin \Sigma$. We conclude that $i+\sigma \in \Sigma$ if and only if $i \in \Sigma$. This implies that $\left(P^{-1}(\alpha)+\sigma\right) \cap \Sigma=\left(P^{-1}(\alpha) \cap \Sigma\right)+\sigma$ and since the set equality $P^{-1}(x+y)=P^{-1}(x)+Y$ holds for any $Y \in P^{-1}(y)$, we get

$$
P^{-1}(\alpha+P(\sigma)) \cap \Sigma=\left(P^{-1}(\alpha)+\sigma\right) \cap \Sigma=\left(P^{-1}(\alpha) \cap \Sigma\right)+\sigma,
$$

which gives a contradiction since $\alpha \in B(\sigma)$. This proves (21).

We recall that the family $\left\{\xi_{j}\right\}$ satisfies the hypothesis $L_{j} \cap \mathbb{Z}^{N}=\{0\}$ for any $j=1, \ldots, K$. Then, setting

$$
r^{j}(\eta)=\inf \left\{\left|i_{j}-i_{j}^{\prime}\right|: i_{j}, i_{j}^{\prime} \in \Lambda_{\eta}^{j}, i_{j} \neq i_{j}^{\prime}\right\}
$$

we show that $\lim _{\eta \rightarrow 0} r^{j}(\eta)=+\infty$ for any $j$. Indeed, suppose by contradiction that there exists a subsequence $\left\{\eta_{k}\right\}$ such that $r^{j}\left(\eta_{k}\right) \leq C_{j}<+\infty$ for any $k$; then for any $k \in \mathbb{N}$ there exist $i_{j}(k)$ and $i_{j}^{\prime}(k)$ in $\Lambda_{\eta_{k}}^{j}$ such that $i_{j}(k) \neq i_{j}^{\prime}(k)$ and $\left|i_{j}(k)-i_{j}^{\prime}(k)\right| \leq C_{j}$. Then (up to subsequences) $i_{j}(k)-i_{j}^{\prime}(k)$ is constant, say $i_{j}(k)-i_{j}^{\prime}(k)=\zeta \in \mathbb{Z}^{N} \backslash\{0\}$. Since $i_{j}(k)$ and $i_{j}^{\prime}(k)$ belong to $\Lambda_{\eta_{k}}^{j}$, it follows that for any $k \operatorname{dist}\left(\zeta, L_{j}\right) \leq 2 \eta_{k} \rightarrow 0$; this implies $\zeta \in L_{j}$, giving the contradiction since $L_{j} \cap \mathbb{Z}^{N}=\{0\}$. Setting

$$
r_{\eta}=\min \left\{r^{j}(\eta): j=1, \ldots, K\right\},
$$

it follows that $\lim _{\eta \rightarrow 0} r_{\eta}=+\infty$, and satisfies the property that if $i$ and $i^{\prime}$ belong to $\Lambda_{\eta}^{j}$ and $\left|i-i^{\prime}\right| \leq r_{\eta}$, then $i=i^{\prime}$. Therefore, for any closed ball $D_{\eta}^{N} \subset \mathbb{R}^{N}$ with diameter $r_{\eta}$ we get

$$
\#\left(D_{\eta}^{N} \cap \Lambda_{\eta}\right) \leq K
$$

Now we define

$$
R_{\eta}=\frac{r_{\eta}}{\sqrt{n}(1+p)}
$$

where

$$
p=\left\|\left(\left.P\right|_{\Pi_{n}}\right)^{-1}\right\|=\max \left\{\left|\left(\left.P\right|_{\Pi_{n}}\right)^{-1}(v)\right|:|v|=1\right\} .
$$

Let $D_{\eta}^{n}$ be the ball with center $x$ and diameter $\sqrt{n} R_{\eta}$; we now prove that

$$
\operatorname{diam}\left(P^{-1}\left(D_{\eta}^{n}\right) \cap S_{\eta}\right) \leq r_{\eta}
$$

Let $c$ be the diameter of $S_{\eta} \cap P^{-1}(0)$. If $i, i^{\prime} \in P^{-1}\left(D_{\eta}^{n}\right) \cap S_{\eta}$, and $w=P\left(i-i^{\prime}\right)$, then $i-i^{\prime}$ admits a decomposition $i-i^{\prime}=\left(\left.P\right|_{\Pi_{n}}\right)^{-1}(w)+w_{0}$, where $w_{0} \in P^{-1}(0)$. We have

$$
\left|i-i^{\prime}\right| \leq c+\left|\left(\left.P\right|_{\Pi_{n}}\right)^{-1}(w)\right| \leq \sqrt{n} R_{\eta}+p|w| \leq \sqrt{n} R_{\eta}(1+p) \leq r_{\eta},
$$


since $c \leq \sqrt{n} R_{\eta}$ for $\eta$ small enough.

If $\alpha \in\left(R_{\eta} Q^{\nu}+x\right) \cap P\left(S_{\eta} \cap \Lambda_{\eta}\right)$, there exists $i_{\alpha} \in P^{-1}(\alpha) \cap S_{\eta} \cap \Lambda_{\eta} \subset P^{-1}\left(D_{\eta}^{n}\right) \cap S_{\eta} \cap \Lambda_{\eta}$; then, (26) implies

$$
\left(R_{\eta} Q^{\nu}+x\right) \cap P\left(S_{\eta} \cap \Lambda_{\eta}\right) \subset P\left(P^{-1}\left(D_{\eta}^{n}\right) \cap S_{\eta} \cap \Lambda_{\eta}\right) \subset P\left(D_{\eta}^{N} \cap \Lambda_{\eta}\right),
$$

where $D_{\eta}^{N}$ denotes a closed ball in $\mathbb{R}^{N}$ with diameter $r_{\eta}$ as in (25). Thus, (21) and (25) give

$$
\#\left(\left(R_{\eta} Q^{\nu}+x\right) \cap B_{\eta}\right) \leq K
$$

and the proof is concluded.

\section{The surface-tension formula}

In this section we prove Proposition 3, which will be obtained using the following result.

Proposition 9. Let $\nu \in S^{n-1}, T>0$, and let $u_{T} \in \mathcal{A}$ satisfy $F\left(u_{T}, \overline{T Q^{\nu}}\right) \leq C T^{n-1}$ and the boundary condition $P u_{T}=v^{\nu}$ on $\mathbb{Z}^{n} \backslash T Q^{\nu}$, where $v^{\nu}$ is defined in (13); then for all $\eta>0$ and for all $\tau \in T_{\eta}^{n}$ there exists $u_{T}^{\tau}$ such that $\left(P u_{T}^{\tau}\right)(\alpha)=v^{\nu}(\alpha-\tau)$ for $\alpha \in \mathbb{Z}^{n} \backslash\left(T Q^{\nu}+\tau\right)$ and

$$
F\left(u_{T}^{\tau}, \overline{T Q^{\nu}+\tau}\right) \leq F\left(u_{T}, \overline{T Q^{\nu}}\right)+\frac{c}{R_{\eta}} T^{n-1},
$$

where $c$ depends only on $n, N, \Sigma$ and $C$, and $R_{\eta}$ is given by Proposition 8.

Proof. The idea behind the construction of $u_{T}^{\tau}$ is that we can simply translate the function $u_{T}$ except close to some set of exceptional points. This exceptional set is not negligible, but the function $u_{T}$ must be constant on some sets enclosing most of such points, so that $u_{T}^{\tau}$ can be defined as that constant in the interior of the translation of those enclosing sets. Proposition 8 allows to quantify this process. In the sequel, $c$ will be used to denote possibly different positive constants depending only on $n, N$ and $\Sigma$, and on the constant $C$ in the hypothesis on the energy $F\left(u_{T}, \overline{T Q^{\nu}}\right)$.

Given $\eta>0$ and $\tau \in T_{\eta}^{n}$, we set (omitting the dependence on $\eta$ ) $\sigma$ as the unique element in $P^{-1}(\tau) \cap T_{\eta}^{N}$ and $I_{T}=\overline{T Q^{\nu}} \cap B(\sigma)$. Thanks to Proposition 8, for any $\gamma \in \mathbb{Z}^{n}$ we have $\#\left(B(\sigma) \cap\left(R_{\eta} Q^{\nu}+\gamma\right)\right) \leq K$; then, since $\overline{T Q^{\nu}} \subset\left(T+R_{\eta}\right) Q^{\nu}$, it follows that

$$
\#\left(I_{T} \backslash\left(T-R_{\eta}\right) Q^{\nu}\right) \leq \frac{c T^{n-1}}{R_{\eta}^{n-1}} .
$$

We set $J_{T}=I_{T} \cap\left(T-R_{\eta}\right) Q^{\nu}$; Proposition 8 ensures that there exist $r_{0}>0$ depending only on $n, N$ and $\Sigma$, and a family $\mathcal{S}_{T}$ of indices such that $J_{T}=\bigcup_{s \in \mathcal{S}_{T}} J_{T}^{s}$ and

1. \# $J_{T}^{s} \leq K$ for any $s \in \mathcal{S}_{T}$

2. the set $U_{T}^{s}=\bigcup_{\alpha \in J_{T}^{s}}\left(r_{0} R_{\eta} Q+\alpha\right)$ is connected for any $s \in \mathcal{S}_{T}$;

3. if $s, s^{\prime} \in \mathcal{S}_{T}$ with $s \neq s^{\prime}$ then $U_{T}^{s} \cap U_{T}^{s^{\prime}}=\emptyset$. 
Conditions 1-3 above express the fact that points in $J_{T}$ can be decomposed into "clusters" of at most $K$ elements, at a distance of order $R_{\eta}$. Moreover, we can assume $r_{0}<1 / 2$, so that for $\eta$ small enough we have

$$
\left(r_{0} R_{\eta} Q+\alpha\right) \cap\left((T+2) Q^{\nu} \backslash(T-2) Q^{\nu}\right)=\emptyset
$$

for any $\alpha \in J_{T}$.

Setting $\widetilde{\mathcal{S}}_{T}=\left\{s \in \mathcal{S}_{T}: F\left(u_{T}, U_{T}^{s}\right) \geq \frac{r_{0} R_{\eta}-1}{2}\right\}$, the hypothesis on $F\left(u_{T}, \overline{T Q^{\nu}}\right)$ gives

$$
\# \widetilde{\mathcal{S}}_{T} \leq \frac{2 C T^{n-1}}{r_{0} R_{\eta}-3}
$$

Then, fixed $s \in \mathcal{S}_{T} \backslash \widetilde{\mathcal{S}}_{T}$, we define

$$
\begin{aligned}
& C_{0}=\bigcup_{\alpha \in J_{T}^{s}}(Q+\alpha) \\
& C_{k}=\bigcup_{\alpha \in J_{T}^{s}}((2 k+1) Q+\alpha) \backslash \bigcup_{\alpha \in J_{T}^{s}}((2 k-1) Q+\alpha) \text { for } k=1, \ldots,\left[\frac{r_{0} R_{\eta}-1}{2}\right] .
\end{aligned}
$$

Since $F\left(u_{T}, U_{T}^{s}\right)<\frac{r_{0} R_{\eta}-1}{2}$, there exists an index $k(s)$ such that $F\left(u_{T}, C_{k(s)}\right)=0$; denoting the connected components of $C_{k(s)}$ by $C_{k(s)}^{m}$, with $m=1, \ldots, M_{k(s)}$, we can write

$$
C_{k(s)}^{m}=\bigcup_{\alpha \in \Gamma_{k(s)}^{m}}((2 k(s)+1) Q+\alpha) \backslash \bigcup_{\alpha \in \Gamma_{k(s)}^{m}}((2 k(s)-1) Q+\alpha)
$$

where the sets $\Gamma_{k(s)}^{m}$ are disjoint and $\bigcup_{m=1}^{M_{k(s)}} \Gamma_{k(s)}^{m}=J_{T}^{s}$ (see Fig. 5). Then, for any $m=$ $1, \ldots, M_{k(s)}, u_{T}$ is constant on $\Sigma \cap P^{-1}\left(C_{k(s)}^{m=1} \cap \cap \mathbb{Z}^{n}\right)$. We denote this constant value by $u_{T}^{s, m}$.

Now, we can construct $u_{T}^{\tau}$. For any $s \in \mathcal{S}_{T} \backslash \widetilde{\mathcal{S}}_{T}$, we define $u_{T}^{\tau}$ in $\Sigma \cap P^{-1}\left(U_{T}^{s}+\tau\right)$ by setting

$$
u_{T}^{\tau}(i)= \begin{cases}u_{T}^{s, m} & \text { if } P(i)-\tau \in \bigcup_{\alpha \in \Gamma_{k(s)}^{m}}((2 k(s)+1) Q+\alpha), m=1, \ldots, M_{k(s)} \\ u_{T}(i-\sigma) & \text { otherwise in } \Sigma \cap P^{-1}\left(U_{T}^{s}+\tau\right) ;\end{cases}
$$

it follows that

$$
F\left(u_{T}^{\tau}, U_{T}^{s}+\tau\right) \leq F\left(u_{T}, U_{T}^{s}\right) .
$$

For $s \in \widetilde{\mathcal{S}}_{T}$, the function $u_{T}^{\tau}$ is given by

$$
u_{T}^{\tau}(i)= \begin{cases}1 & \text { if } P(i)-\tau \in B(\sigma) \\ u_{T}(i-\sigma) & \text { otherwise in } \Sigma \cap P^{-1}\left(U_{T}^{s}+\tau\right) .\end{cases}
$$




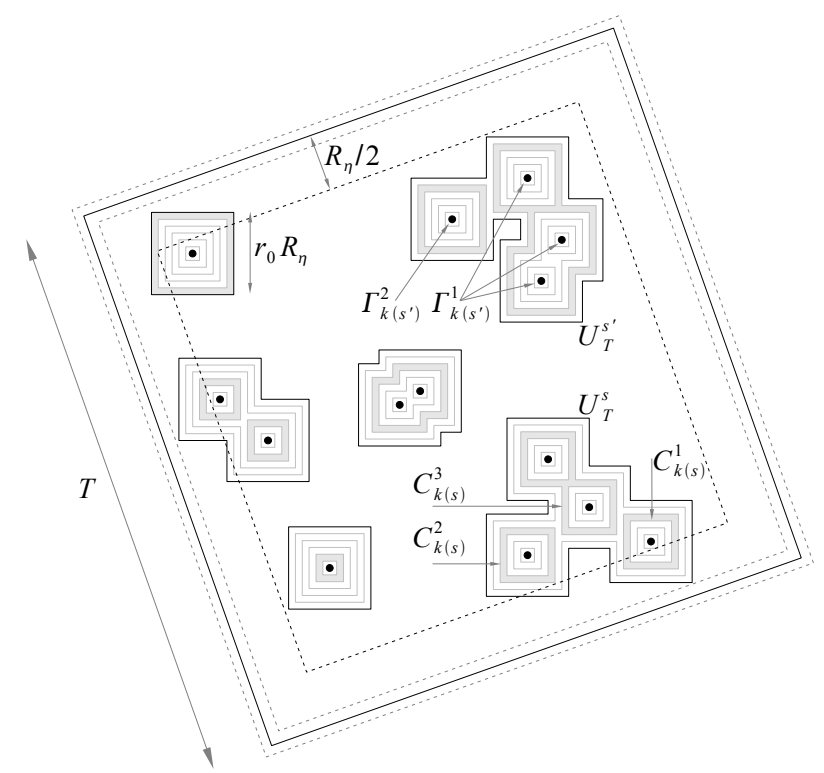

Figure 5: Construction of functions close to exceptional points

Finally, we set

$$
u_{T}^{\tau}(i)= \begin{cases}v^{\nu}(P(i)-\tau) & \text { if } P(i)-\tau \in I_{T} \backslash J_{T} \\ u_{T}(i-\sigma) & \text { otherwise in } \Sigma .\end{cases}
$$

Note that $P\left(u_{T}^{\tau}\right)(\alpha)=v^{\nu}(\alpha-\tau)$ for $\alpha \in \mathbb{Z}^{n} \backslash\left(T Q^{\nu}+\tau\right)$. Recalling (27), (28) and (29) we get

$$
\begin{aligned}
F\left(u_{T}^{\tau}, \overline{T Q^{\nu}+\tau}\right) & \leq F\left(u_{T}, \overline{T Q^{\nu}}\right)+c \#\left(I_{T} \backslash J_{T}\right)+c K \# \widetilde{\mathcal{S}}_{T} \\
& \leq F\left(u_{T}, \overline{T Q^{\nu}}\right)+\frac{c}{R_{\eta}} T^{n-1},
\end{aligned}
$$

as desired.

Remark 10. The same argument as above shows that, given $\tau \in T_{\eta}^{n}$ and a function $u \in \mathcal{A}$ satisfying $F\left(u, \overline{T Q^{\nu}+\tau}\right) \leq C T^{n-1}$ and the boundary condition $(P u)(\alpha)=v^{\nu}(\alpha-\tau)$ for $\alpha \in \mathbb{Z}^{n} \backslash\left(T Q^{\nu}+\tau\right)$, we can construct a function $\widetilde{u} \in \mathcal{A}$ such that $P \widetilde{u}=v^{\nu}$ in $\mathbb{Z}^{n} \backslash T Q^{\nu}$ and

$$
F\left(\widetilde{u}, \overline{T Q^{\nu}}\right) \leq F\left(u, \overline{T Q^{\nu}+\tau}\right)+\frac{c}{R_{\eta}} T^{n-1},
$$

where $R_{\eta}$ is given by Proposition 8 . 
Proof of Proposition 3. We consider $S \gg T$ and the $n$-cube $S Q^{\nu}$. With fixed $\eta>0$, let $L_{\eta}$ be the inclusion length given by the relative density of $T_{\eta}^{n}$, and denote by $Q_{k}^{\nu}$ with $k=1, \ldots,\left[\frac{S}{T+2 L_{\eta}}\right]^{n-1}$ disjoint cubes with two faces orthogonal to $\nu$ and side length $T+2 L_{\eta}$ included in $S Q^{\nu} \cap\left\{|\langle x, \nu\rangle|<\frac{T}{2}+L_{\eta}\right\}$.

The relative density ensures that for any $k$ there exists a translation vector $\tau_{\eta}^{k} \in T_{\eta}^{n}$ such that $T Q^{\nu}+\tau_{\eta}^{k} \subset Q_{k}^{\nu}$.

Following Proposition 9, given $u_{T}$, for any translation vector $\tau_{\eta}^{k}$ we define $u^{k}=u_{T}^{\tau_{\eta}^{k}}$. We then define $u_{S}$ as

$$
u_{S}(i)= \begin{cases}u^{k}(i) & \text { if } P(i) \in T Q^{\nu}+\tau_{\eta}^{k} \\ v^{\nu}(P(i)) & \text { if } P(i) \in \overline{S Q^{\nu}} \backslash \bigcup_{k}\left(T Q^{\nu}+\tau_{\eta}^{k}\right) .\end{cases}
$$

In Fig. 6 we picture the construction of $u_{S}$ by representing the corresponding projections $P\left(u_{S}\right)$ (gray and white regions corresponding to \pm 1 , the 0 zones being negligible).

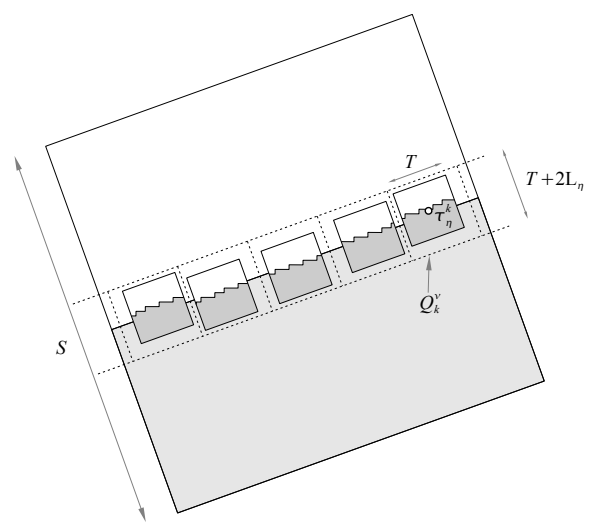

Figure 6: Representation of the construction of $u_{S}$

The contributions to $F\left(u_{S}, \overline{S Q^{\nu}}\right)$ outside the union of the squares $Q_{k}^{\nu}$ can be easily estimated by $c\left(T+2 L_{\eta}\right)^{n-1} S^{n-2}$ since there we have $u_{S}(i)=v^{\nu}(P(i))$.

Hence, the function $u_{S}: \Sigma \cap P^{-1}\left(\overline{S Q^{\nu}}\right) \rightarrow\{-1,+1\}$ defined in (30) satisfies the boundary condition $P u_{S}=v^{\nu}$ in $\overline{S Q^{\nu}} \backslash S Q^{\nu}$ and thanks to Proposition 9

$$
\begin{aligned}
F\left(u_{S}, \overline{S Q^{\nu}}\right) \leq & {\left[\frac{S}{T+2 L_{\eta}}\right]^{n-1}\left(F\left(u_{T}, \overline{T Q^{\nu}}\right)+c \frac{T^{n-1}}{R_{\eta}}+c L_{\eta}^{n-1}\right) } \\
& +c\left(T+2 L_{\eta}\right)^{n-1} S^{n-2} .
\end{aligned}
$$


Thus, taking the upper limit as $S \rightarrow+\infty$,

$$
\begin{aligned}
\limsup _{S \rightarrow+\infty} \frac{1}{S^{n-1}} F\left(u_{S}, \overline{S Q^{\nu}}\right) & \leq \frac{1}{\left(T+2 L_{\eta}\right)^{n-1}}\left(F\left(u_{T}, \overline{T Q^{\nu}}\right)+c \frac{T^{n-1}}{R_{\eta}}+c L_{\eta}^{n-1}\right) \\
& \leq \frac{1}{T^{n-1}}\left(F\left(u_{T}, \overline{T Q^{\nu}}\right)+c \frac{T^{n-1}}{R_{\eta}}+c L_{\eta}^{n-1}\right)
\end{aligned}
$$

and, taking the lower limit as $T \rightarrow+\infty$,

$$
\limsup _{S \rightarrow+\infty} \frac{1}{S^{n-1}} F\left(u_{S}, \overline{S Q^{\nu}}\right) \leq \liminf _{T \rightarrow+\infty} \frac{1}{T^{n-1}} F\left(u_{T}, \overline{T Q^{\nu}}\right)+\frac{c}{R_{\eta}} .
$$

Now, for a given $r>0$, let $u_{T}^{r}: \Sigma \rightarrow\{-1,+1\}$ satisfy $P u_{T}^{r}=v^{\nu}$ in $\mathbb{Z}^{n} \backslash T Q^{\nu}$ and be such that

$$
F\left(u_{T}^{r}, \overline{T Q^{\nu}}\right) \leq g_{T}(\nu)+r .
$$

Then $u_{T}^{r}$ satisfies the hypothesis of Proposition 9, and

$$
\limsup _{S \rightarrow+\infty} \frac{1}{S^{n-1}} g_{S}(\nu) \leq \liminf _{T \rightarrow+\infty} \frac{1}{T^{n-1}} g_{T}(\nu)+\frac{c}{R_{\eta}}
$$

Letting $\eta \rightarrow 0$, since $R_{\eta} \rightarrow+\infty$ by Proposition 8 , we have

$$
\limsup _{S \rightarrow+\infty} \frac{1}{S^{n-1}} g_{S}(\nu) \leq \liminf _{T \rightarrow+\infty} \frac{1}{T^{n-1}} g_{T}(\nu)
$$

and we conclude that the limit $\varphi(\nu)=\lim _{T \rightarrow+\infty} \frac{1}{T^{n-1}} g_{T}(\nu)$ exists.

\section{$5 \quad$ Homogenization of quasicrystalline lattices}

In this Section we prove Theorem 4 by showing separately a lower and an upper bound.

\subsection{Lower bound}

Let $A$ be a set of finite perimeter in $\mathbb{R}^{n}$ and let $\left\{u_{\varepsilon}\right\}$ be a sequence of admissible functions such that $u_{\varepsilon} \rightarrow A$, where the convergence is as in (12). It is not restrictive to assume that there exists the limit $\lim _{\varepsilon \rightarrow 0^{+}} F_{\varepsilon}\left(u_{\varepsilon}\right)$ and that it is finite.

We define the measures

$$
\begin{aligned}
& \mu_{\varepsilon}=\varepsilon^{n-1} \sum_{\alpha \in \mathbb{Z}^{n}} \delta_{\alpha} \#\left\{\{i, j\} \in N\left(\left\{P^{-1}(\alpha) \cap \Sigma\right\}\right): u_{\varepsilon}(i) \neq u_{\varepsilon}(j)\right\} \\
& +\varepsilon^{n-1} \sum_{\{\alpha, \beta\} \in N\left(\mathbb{Z}^{n}\right)} \delta_{\frac{\alpha+\beta}{2}} \#\left\{\{i, j\} \in N(\Sigma): i \in P^{-1}(\alpha), j \in P^{-1}(\beta), u_{\varepsilon}(i) \neq u_{\varepsilon}(j)\right\},
\end{aligned}
$$

where for $E \subset \mathbb{Z}^{n}$ (or $\mathbb{Z}^{N}$ ) we set $N(E)=\{\{\alpha, \beta\}: \alpha, \beta \in E,|\alpha-\beta|=1\}$. In this way, $\mu_{\varepsilon}\left(\mathbb{R}^{n}\right)=F_{\varepsilon}\left(u_{\varepsilon}\right)$, so that the sequence $\left\{\mu_{\varepsilon}\right\}$ is bounded, and, up to subsequences, we can assume $\mu_{\varepsilon} \stackrel{*}{\rightarrow} \mu$. 
We want to prove that the inequality

$$
\frac{d \mu}{d \mathcal{H}^{n-1}\left\llcorner\partial^{*} A\right.}\left(x_{0}\right) \geq \varphi\left(\nu_{A}\left(x_{0}\right)\right)
$$

holds for $\mathcal{H}^{n-1}$-a.a. $x_{0} \in \partial^{*} A$.

With fixed $x_{0} \in \partial^{*} A$ we set $\nu=\nu_{A}\left(x_{0}\right)$, and consider as above $\varrho Q^{\nu}+x_{0}$ as any cube in $\mathbb{R}^{n}$ with centre $x_{0}$, side length $\varrho$ and two faces orthogonal to $\nu$. Note that, for almost every $\varrho, \mu\left(\varrho Q^{\nu}+x_{0}\right)=\lim _{\varepsilon \rightarrow 0} \mu_{\varepsilon}\left(\varrho Q^{\nu}+x_{0}\right)$.

By the Besicovitch differentiation theorem it follows that $\mathcal{H}^{n-1}$-almost every $x_{0} \in \partial^{*} A$ is a Lebesgue point for $\mu$ with respect to $\mathcal{H}^{n-1}\left\llcorner\partial^{*} A\right.$. Hence, for $\mathcal{H}^{n-1}$-a.a. $x_{0} \in \partial^{*} A$ there exists the limit

$$
\frac{d \mu}{d \mathcal{H}^{n-1}\left\llcorner\partial^{*} A\right.}\left(x_{0}\right)=\lim _{\varrho \rightarrow 0} \frac{\mu\left(\varrho Q^{\nu}+x_{0}\right)}{\mathcal{H}^{n-1}\left(\left(\varrho Q^{\nu}+x_{0}\right) \cap \partial^{*} A\right)} .
$$

For $\mathcal{H}^{n-1}$-a.a. $x_{0} \in \partial^{*} A$ we have $\frac{A-x_{0}}{\varrho} \rightarrow\{x:\langle x, \nu\rangle \geq 0\}$ as $\varrho \rightarrow 0$.

We may therefore assume that $x_{0} \in \partial^{*} A$ satisfies the properties above.

Since $\mu$ is finite it is also possible to choose an infinitesimal sequence $\varrho_{\varepsilon}$ such that

$$
\frac{d \mu}{d \mathcal{H}^{n-1}\left\llcorner\partial^{*} A\right.}\left(x_{0}\right)=\liminf _{\varepsilon \rightarrow 0} \frac{\mu_{\varepsilon}\left(\varrho_{\varepsilon} Q^{\nu}+x_{0}\right)}{\mathcal{H}^{n-1}\left(\left(\varrho_{\varepsilon} Q^{\nu}+x_{0}\right) \cap \partial^{*} A\right)},
$$

satisfying the asymptotic conditions $T_{\varepsilon}=\frac{\varrho_{\varepsilon}}{\varepsilon} \rightarrow+\infty$ and, setting $x_{\varepsilon}=\frac{x_{0}}{\varepsilon}$

$$
\frac{1}{T_{\varepsilon}^{n}} \int_{T_{\varepsilon} Q^{\nu}+x_{\varepsilon}}\left|v_{\varepsilon}(\varepsilon x)-v^{\nu}\left(x-x_{\varepsilon}\right)\right| d x=o(1)_{\varepsilon \rightarrow 0} .
$$

Then we get

$$
\begin{aligned}
\liminf _{\varepsilon \rightarrow 0} \frac{\mu_{\varepsilon}\left(\varrho_{\varepsilon} Q^{\nu}+x_{0}\right)}{\mathcal{H}^{n-1}\left(\left(\varrho_{\varepsilon} Q^{\nu}+x_{0}\right) \cap \partial^{*} A\right)} & =\liminf _{\varepsilon \rightarrow 0} \frac{F_{\varepsilon}\left(u_{\varepsilon}, \varrho_{\varepsilon} Q^{\nu}+x_{0}\right)}{\varrho_{\varepsilon}^{n-1}} \\
& =\liminf _{\varepsilon \rightarrow 0} \frac{F\left(w_{\varepsilon}, T_{\varepsilon} Q^{\nu}+x_{\varepsilon}\right)}{T_{\varepsilon}^{n-1}}
\end{aligned}
$$

where $w_{\varepsilon}(i)=u_{\varepsilon}(\varepsilon i)$ in $\Sigma$ so that $P w_{\varepsilon}(\alpha)=v_{\varepsilon}(\varepsilon \alpha)$.

The relative density of the set of translations $T_{\eta}^{n}$ ensures that fixed $\eta>0$ there exists $\tau^{\varepsilon} \in T_{\eta}^{n}$ such that $\left(T_{\varepsilon}-2 L_{\eta}\right) Q^{\nu}+\tau^{\varepsilon} \subset T_{\varepsilon} Q^{\nu}+x_{\varepsilon}$. Note that

$$
\int_{\left(T_{\varepsilon}-2 L_{\eta}\right) Q^{\nu}+\tau^{\varepsilon}}\left|v^{\nu}\left(x-x_{\varepsilon}\right)-v^{\nu}\left(x-\tau^{\varepsilon}\right)\right| d x=O\left(T_{\varepsilon}^{n-1}\right)_{\varepsilon \rightarrow 0} .
$$

We set $\widetilde{T}_{\varepsilon}=T_{\varepsilon}-2 L_{\eta}$; from (32) we get

$$
\frac{1}{T_{\varepsilon}^{n}} \int_{\widetilde{T}_{\varepsilon} Q^{\nu}+\tau^{\varepsilon}}\left|v_{\varepsilon}(\varepsilon x)-v^{\nu}\left(x-\tau^{\varepsilon}\right)\right| d x=o(1)_{\varepsilon \rightarrow 0} .
$$


Now, we fix $\delta \in(0,1 / 4)$ and define

$$
Q^{\nu}(m, \varepsilon)=\left(\widetilde{T}_{\varepsilon}-2\left(\delta \widetilde{T}_{\varepsilon}+3 m \sqrt{n}\right)\right) Q^{\nu}+\tau_{\varepsilon}, \quad m=0, \ldots, N_{\varepsilon}=\left[\frac{\delta \widetilde{T}_{\varepsilon}}{3 \sqrt{n}}\right] .
$$

Moreover, we set $C(m, \varepsilon)=Q^{\nu}(m-1, \varepsilon) \backslash Q^{\nu}(m, \varepsilon)$. The definition of $Q^{\nu}(m, \varepsilon)$ is envisaged so that the cube $Q^{\nu}(m, \varepsilon)$ is well separated from the exterior of $Q^{\nu}(m-1, \varepsilon)$ (see also Fig. 7).

Then there exists $m(\varepsilon) \in\left\{1, \ldots, N_{\varepsilon}\right\}$ such that

$$
\begin{aligned}
\mid\left\{x \in \widetilde{T}_{\varepsilon} Q^{\nu}\right. & \left.+\tau^{\varepsilon}: P w_{\varepsilon}(x) \neq v^{\nu}\left(x-\tau^{\varepsilon}\right)\right\} \mid \\
& \geq \sum_{m=1}^{N_{\varepsilon}} \#\left\{\alpha \in \mathbb{Z}^{n}: Q+\alpha \subset C(m, \varepsilon) \text { and } P w_{\varepsilon}(\alpha) \neq v^{\nu}\left(\alpha-\tau^{\varepsilon}\right)\right\} \\
& \geq \#\left\{\alpha \in \mathbb{Z}^{n}: Q+\alpha \subset C(m(\varepsilon), \varepsilon) \text { and } P w_{\varepsilon}(\alpha) \neq v^{\nu}\left(\alpha-\tau^{\varepsilon}\right)\right\} N_{\varepsilon}
\end{aligned}
$$

so that recalling (33) we get

$$
\#\left\{\alpha \in \mathbb{Z}^{n}: Q+\alpha \subset C(m(\varepsilon), \varepsilon) \text { and } P w_{\varepsilon}(\alpha) \neq v^{\nu}\left(\alpha-\tau^{\varepsilon}\right)\right\}=o\left(\widetilde{T}_{\varepsilon}^{n-1}\right)_{\varepsilon \rightarrow 0} .
$$

Now we define $\widetilde{w}_{\varepsilon}: \Sigma \rightarrow\{-1,+1\}$ as

$$
\widetilde{w}_{\varepsilon}(i)= \begin{cases}w_{\varepsilon}(i) & \text { if }(Q+P(i)) \cap Q^{\nu}(m(\varepsilon)+1, \varepsilon) \neq \emptyset \\ v^{\nu}\left(P(i)-\tau^{\varepsilon}\right) & \text { otherwise }\end{cases}
$$

(see Fig. 7).
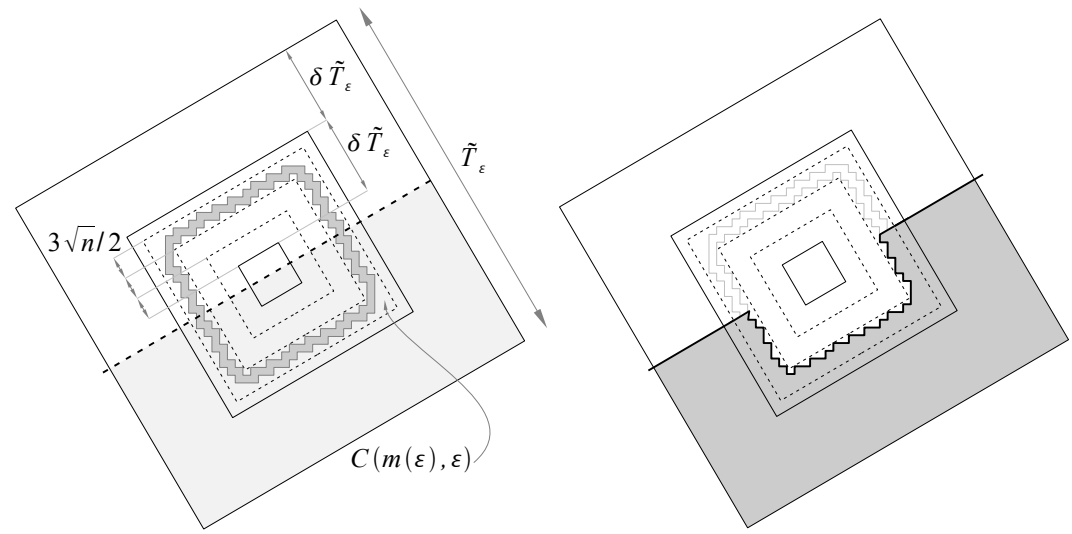

Figure 7: Modification of boundary values

Recalling (34), it follows that $\widetilde{w}_{\varepsilon}$ satisfies the boundary conditions, and by construction

$$
F\left(\widetilde{w}_{\varepsilon}, \overline{\widetilde{T}_{\varepsilon}} Q^{\nu}+\tau_{\varepsilon}\right) \leq F\left(w_{\varepsilon}, \widetilde{T}_{\varepsilon} Q^{\nu}+\tau_{\varepsilon}\right)+o\left(\widetilde{T}_{\varepsilon}^{n-1}\right)_{\varepsilon \rightarrow 0}+c \widetilde{T}_{\varepsilon}^{n-1} \delta .
$$


Taking the liminf as $\varepsilon \rightarrow 0$, and the limit for $\delta \rightarrow 0$, the previous inequality implies

$$
\liminf _{\varepsilon \rightarrow 0} \frac{F\left(\widetilde{w}_{\varepsilon}, \overline{\widetilde{T}_{\varepsilon} Q^{\nu}+\tau_{\varepsilon}}\right)}{\widetilde{T}_{\varepsilon}^{n-1}} \leq \liminf _{\varepsilon \rightarrow 0} \frac{F\left(w_{\varepsilon}, \widetilde{T}_{\varepsilon} Q^{\nu}+\tau_{\varepsilon}\right)}{\widetilde{T}_{\varepsilon}^{n-1}} .
$$

Now, Remark 10 ensures that there exists $\widehat{w}_{\varepsilon} \in \mathcal{A}$ with $P \widehat{w}_{\varepsilon}=v^{\nu}$ in $\mathbb{Z}^{n} \backslash \widetilde{T}_{\varepsilon} Q^{\nu}$ such that

$$
F\left(\widehat{w}_{\varepsilon}, \overline{\widetilde{T}_{\varepsilon} Q^{\nu}}\right) \leq F\left(\widetilde{w}_{\varepsilon}, \overline{\widetilde{T}_{\varepsilon} Q^{\nu}+\tau^{\varepsilon}}\right)+\frac{c}{R_{\eta}} \widetilde{T}_{\varepsilon}^{n-1},
$$

and we can conclude

$$
\begin{aligned}
\liminf _{\varepsilon \rightarrow 0} \frac{\mu_{\varepsilon}\left(\varrho_{\varepsilon} Q^{\nu}+x_{0}\right)}{\mathcal{H}^{n-1}\left(\left(\varrho_{\varepsilon} Q^{\nu}+x_{0}\right) \cap \partial^{*} A\right)} \geq \liminf _{\varepsilon \rightarrow 0} \frac{F\left(w_{\varepsilon}, \widetilde{T}_{\varepsilon} Q^{\nu}+\tau^{\varepsilon}\right)}{\widetilde{T}_{\varepsilon}^{n-1}} \\
\geq \liminf _{\varepsilon \rightarrow 0} \frac{F\left(\widetilde{w}_{\varepsilon}, \widetilde{T}_{\varepsilon} Q^{\nu}+\tau_{\varepsilon}\right)}{\widetilde{T}_{\varepsilon}^{n-1}} \geq \liminf _{\varepsilon \rightarrow 0} \frac{F\left(\widehat{w}_{\varepsilon}, \widetilde{T}_{\varepsilon} Q^{\nu}\right)}{\widetilde{T}_{\varepsilon}^{n-1}}-\frac{c}{R_{\eta}} .
\end{aligned}
$$

Since $R_{\eta} \rightarrow+\infty$ as $\eta \rightarrow 0$, Proposition 3 finally allows to conclude that

$$
\liminf _{\varepsilon \rightarrow 0} \frac{\mu_{\varepsilon}\left(\varrho_{\varepsilon} Q^{\nu}+x_{0}\right)}{\mathcal{H}^{n-1}\left(\left(\varrho_{\varepsilon} Q^{\nu}+x_{0}\right) \cap \partial^{*} A\right)} \geq \varphi(\nu)
$$

\subsection{Upper bound}

The proof of the upper bound will use a density argument, following the line of the proof of the upper bound in [11]. We start by proving in Proposition 11 the existence of a recovery sequence when $A$ is a polyhedral set.

Proposition 11. Let $A \subset \mathbb{R}^{n}$ be a polyhedral set. Then there exists a sequence $\left\{u_{\varepsilon}\right\}$, $u_{\varepsilon} \in \mathcal{A}_{\varepsilon}$ such that $u_{\varepsilon} \rightarrow A$ as $\varepsilon \rightarrow 0$ (where the convergence is given by (12)) and

$$
\limsup _{\varepsilon \rightarrow 0} F_{\varepsilon}\left(u_{\varepsilon}\right) \leq F_{0}(A)=\int_{\partial A} \varphi(\nu) d \mathcal{H}^{n-1} .
$$

Proof. Fixed $r>0$, omitting the dependence on $r$, for any $S>0$ we denote by $u_{S}^{\nu} \in \mathcal{A}$ a function satisfying $P u_{S}^{\nu}=v^{\nu}$ in $\mathbb{Z}^{n} \backslash S Q^{\nu}$ and

$$
F\left(u_{S}^{\nu}, \overline{S Q^{\nu}}\right)<g_{S}(\nu)+r .
$$

Our construction is localized close to each $(n-1)$-dimensional face of the polyhedral set $A$. We start by considering, for $\bar{x} \in \mathbb{R}^{n}$ and $\nu \in S^{n-1}$, the affine space $L=\{\langle x-\bar{x}, \nu\rangle=0\}$. Let $E$ be a $(n-1)$-dimensional polyhedral subset of $L$.

Fixed $\varepsilon>0$ small enough, we denote by $Q_{\varepsilon}^{k}=\sqrt{\varepsilon} Q^{\nu}+x_{\varepsilon}^{k}$, for $k=1, \ldots, M_{\varepsilon}$, each cube of a maximal family of disjoint cubes with center in $E$, two faces orthogonal to $\nu$ and side length $\sqrt{\varepsilon}$, such that $Q_{\varepsilon}^{k} \cap L \subset E$. Note that

$$
M_{\varepsilon}=\mathcal{H}^{n-1}(E)(\sqrt{\varepsilon})^{1-n}+o\left((\sqrt{\varepsilon})^{1-n}\right)_{\varepsilon \rightarrow 0} .
$$


Setting

$$
E_{\lambda}=\{x+t \nu: x \in E,-\lambda<t<\lambda\}
$$

for $\lambda>0$, we can estimate

$$
\#\left(\varepsilon \mathbb{Z}^{n} \cap\left(E_{2 \varepsilon \sqrt{n}} \backslash \bigcup_{k} Q_{\varepsilon}^{k}\right)\right) \leq c \sqrt{\varepsilon} \varepsilon^{1-n} .
$$

For any $\eta>0$, the relative density of $T_{\eta}^{n}$ ensures that for any $\varepsilon>0$ and $k$ there exists $\tau_{\varepsilon}^{k} \in T_{\eta}^{n}$ (where we omit the dependence on $\eta$ ) such that $\varepsilon^{-1} x_{\varepsilon}^{k} \in\left[0, L_{\eta}\right]^{n}+\tau_{\varepsilon}^{k}$; hence, for $T$ large enough

$$
\overline{\left(T-4 L_{\eta}\right) Q^{\nu}+\tau_{\varepsilon}^{k}} \subset T Q^{\nu}+\varepsilon^{-1} x_{\varepsilon}^{k}
$$

so that, choosing $T=1 / \sqrt{\varepsilon}$

$$
\overline{\left(\sqrt{\varepsilon}-4 \varepsilon L_{\eta}\right) Q^{\nu}+\varepsilon \tau_{\varepsilon}^{k}} \subset \sqrt{\varepsilon} Q^{\nu}+x_{\varepsilon}^{k} .
$$

Setting $\varrho_{\varepsilon}=\sqrt{\varepsilon}-4 \varepsilon L_{\eta}$ (again omitting the dependence on $\eta$ ), Proposition 9 ensures that for each $\varepsilon>0$ and $k=1, \ldots, M_{\varepsilon}$ there exists $u_{\varepsilon}^{k} \in \mathcal{A}$ such that $P\left(u_{\varepsilon}^{k}\right)(\alpha)=v^{\nu}\left(\alpha-\tau_{\varepsilon}^{k}\right)$ in $\mathbb{Z}^{n} \backslash\left(\left(\varrho_{\varepsilon} / \varepsilon\right) Q^{\nu}+\tau_{\varepsilon}^{k}\right)$ and

$$
F\left(u_{\varepsilon}^{k}, \overline{\left(\varrho_{\varepsilon} / \varepsilon\right) Q^{\nu}+\tau_{\varepsilon}^{k}}\right) \leq F\left(u_{\varrho_{\varepsilon} / \varepsilon}^{\nu}, \overline{\left(\varrho_{\varepsilon} / \varepsilon\right) Q^{\nu}}\right)+\frac{c}{R_{\eta}}\left(\frac{\varrho_{\varepsilon}}{\varepsilon}\right)^{n-1}
$$

where $u_{\varrho_{\varepsilon} / \varepsilon}^{\nu}$ is as in (36) with $S=\varrho_{\varepsilon} / \varepsilon$. Defining for each $k=1, \ldots, M_{\varepsilon}$ the function $w_{\varepsilon}^{k} \in \mathcal{A}_{\varepsilon}$ as $w_{\varepsilon}^{k}(\varepsilon i)=u_{\varepsilon}^{k}(i)$, it follows that

$$
\begin{aligned}
F_{\varepsilon}\left(w_{\varepsilon}^{k}, \overline{\varrho_{\varepsilon} Q^{\nu}+\varepsilon \tau_{\varepsilon}^{k}}\right) & =\varepsilon^{n-1} F\left(u_{\varepsilon}^{k}, \overline{\left(\varrho_{\varepsilon} / \varepsilon\right) Q^{\nu}+\tau_{\varepsilon}^{k}}\right) \\
& \leq \varepsilon^{n-1} F\left(u_{\varrho_{\varepsilon} / \varepsilon}^{\nu}, \overline{\left(\varrho_{\varepsilon} / \varepsilon\right) Q^{\nu}}\right)+\frac{c}{R_{\eta}} \varrho_{\varepsilon}^{n-1} \\
& \leq \varepsilon^{n-1} g_{\varrho_{\varepsilon} / \varepsilon}(\nu)+\varepsilon^{n-1} r+\frac{c}{R_{\eta}} \varrho_{\varepsilon}^{n-1}
\end{aligned}
$$

Now, for a fixed $\lambda>\sqrt{\varepsilon}$, we define $w_{\varepsilon}: \varepsilon \Sigma \cap P^{-1}\left(E_{\lambda}\right) \rightarrow\{-1,+1\}$ as

$$
w_{\varepsilon}(\varepsilon i)= \begin{cases}w_{\varepsilon}^{k}(\varepsilon i) & \text { if } P(\varepsilon i) \in \overline{\varrho_{\varepsilon} Q^{\nu}+\varepsilon \tau_{\varepsilon}^{k}} \\ v_{\varepsilon}^{\nu}(P(\varepsilon i)) & \text { otherwise. }\end{cases}
$$

where

$$
v_{\varepsilon}^{\nu}(\varepsilon \alpha)= \begin{cases}1 & \text { if } \varepsilon \alpha \in\{x+t \nu, x \in E, 0 \leq t<\lambda\} \\ -1 & \text { if } \varepsilon \alpha \in\{x+t \nu, x \in E,-\lambda<t<0\} .\end{cases}
$$

In Fig. 8 we picture the construction of $w_{\varepsilon}$ by representing the corresponding projections $P\left(w_{\varepsilon}\right)$.

Since for each $k$ we have

$$
\#\left(\varepsilon \mathbb{Z}^{n} \cap E_{2 \varepsilon \sqrt{n}} \cap\left(Q_{\varepsilon}^{k} \backslash \overline{\varrho_{\varepsilon} Q^{\nu}+\varepsilon \tau_{\varepsilon}^{k}}\right)\right) \leq c(\sqrt{\varepsilon})^{2-n},
$$




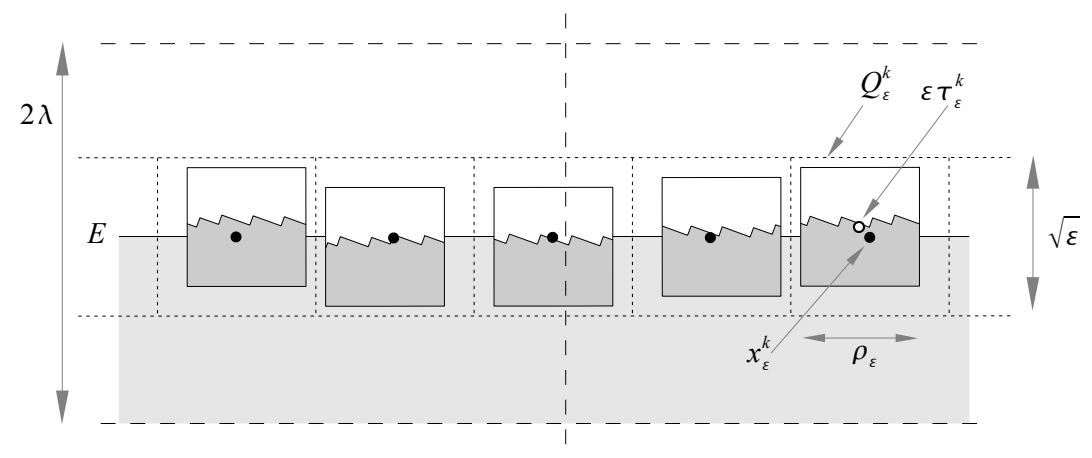

Figure 8: Construction of $w_{\varepsilon}$

recalling (37) we have

$$
\begin{aligned}
F_{\varepsilon}\left(w_{\varepsilon}, E_{\lambda}\right) & \leq \sum_{k} F_{\varepsilon}\left(w_{\varepsilon}^{k}, \overline{\varrho_{\varepsilon} Q^{\nu}+\varepsilon \tau_{\varepsilon}^{k}}\right)+c \varepsilon^{n-1} \#\left(\varepsilon \mathbb{Z}^{n} \cap\left(E_{2 \varepsilon \sqrt{n}} \backslash \bigcup_{k} \overline{\varrho_{\varepsilon} Q^{\nu}+\varepsilon \tau_{\varepsilon}^{k}}\right)\right) \\
& \leq M_{\varepsilon}\left(\varepsilon^{n-1} g_{\varrho_{\varepsilon} / \varepsilon}(\nu)+\varepsilon^{n-1} r+\frac{c}{R_{\eta}} \varrho_{\varepsilon}^{n-1}\right)+c \sqrt{\varepsilon} \\
& \leq \mathcal{H}^{n-1}(E)(\sqrt{\varepsilon})^{n-1}\left(g_{\varrho_{\varepsilon} / \varepsilon}(\nu)+r\right)+\frac{c}{R_{\eta}}\left(\frac{\varrho_{\varepsilon}}{\sqrt{\varepsilon}}\right)^{n-1}+o(1)_{\varepsilon \rightarrow 0} \\
& \leq \mathcal{H}^{n-1}(E)\left(\frac{\varepsilon}{\varrho_{\varepsilon}}\right)^{n-1}\left(g_{\varrho_{\varepsilon} / \varepsilon}(\nu)+r\right)+\frac{c}{R_{\eta}}+o(1)_{\varepsilon \rightarrow 0}
\end{aligned}
$$

since $\varrho_{\varepsilon}<\sqrt{\varepsilon}$ and $\lim _{\varepsilon \rightarrow 0} \frac{\varrho_{\varepsilon}}{\sqrt{\varepsilon}}=1$. Then

$$
\begin{aligned}
\limsup _{\varepsilon \rightarrow 0} F_{\varepsilon}\left(w_{\varepsilon}, E_{\lambda}\right) & \leq \mathcal{H}^{n-1}(E) \limsup _{\varepsilon \rightarrow 0} \frac{1}{\left(\varrho_{\varepsilon} / \varepsilon\right)^{n-1}} g_{\varrho_{\varepsilon} / \varepsilon}(\nu)+\frac{c}{R_{\eta}} \\
& =\mathcal{H}^{n-1}(E) \varphi(\nu)+\frac{c}{R_{\eta}}
\end{aligned}
$$

Since $R_{\eta} \rightarrow+\infty$ as $\eta \rightarrow 0$, we conclude

$$
\limsup _{\varepsilon \rightarrow 0} F_{\varepsilon}\left(w_{\varepsilon}, E_{\lambda}\right) \leq \mathcal{H}^{n-1}(E) \varphi(\nu) .
$$

Now, let $A$ be a polyhedral set in $\mathbb{R}^{n}$ with $(n-1)$-dimensional faces $E^{1}, \ldots, E^{q}$. We denote by $\nu^{j}$ the inner normal to $E^{j}$ and by $E_{\lambda}^{j}$ the set $\left\{x+t \nu^{j}: x \in E,-\lambda<t<\lambda\right\}$.

Let $w_{\varepsilon, j}: \varepsilon \Sigma \cap P^{-1}\left(E_{\lambda}^{j}\right) \rightarrow\{-1,+1\}$ be the function constructed as in the previous step, by choosing $E=E^{j}, \nu=\nu^{j}$ and $E_{\lambda}=E_{\lambda}^{j}$. Now, we define

$$
u_{\varepsilon}(\varepsilon i)= \begin{cases}w_{\varepsilon, j}(\varepsilon i) & \text { if } P(\varepsilon i) \in E_{\sqrt{\varepsilon}}^{j} \backslash \bigcup_{k \neq l}\left(E_{\sqrt{\varepsilon}}^{k} \cap E_{\sqrt{\varepsilon}}^{l}\right) \\ \chi_{A \cap \varepsilon \mathbb{Z}^{n}}(P(\varepsilon i)) & \text { otherwise in } \varepsilon \Sigma .\end{cases}
$$


With this definition, $u_{\varepsilon} \rightarrow A$ in the sense of (12); since $\left|\bigcup_{k \neq l}\left(E_{\sqrt{\varepsilon}}^{k} \cap E_{\sqrt{\varepsilon}}^{l}\right)\right| \rightarrow 0$ as $\varepsilon \rightarrow 0$, recalling (39) we get

$$
\limsup _{\varepsilon \rightarrow 0} F_{\varepsilon}\left(u_{\varepsilon}\right) \leq \sum_{j} \mathcal{H}^{n-1}\left(E^{j}\right) \varphi\left(\nu^{j}\right)=\int_{\partial A} \varphi(\nu) d \mathcal{H}^{n-1}=F_{0}(A) .
$$

concluding the proof.

To treat the case of a general $A$, we use a density argument based on the Reshetnjak Theorem. Given $A$ with finite perimeter in $\mathbb{R}^{n}$, let $\left\{A_{k}\right\}$ be a sequence of polyhedral sets such that $\left|A \Delta A_{k}\right| \rightarrow 0$ and $\left|D \chi_{A_{k}}\right|\left(\mathbb{R}^{n}\right) \rightarrow\left|D \chi_{A}\right|\left(\mathbb{R}^{n}\right)$ as $k \rightarrow+\infty$ (see [3]). The same proof as $[11$, Sec. 4.2$]$ shows that the function $\varphi$ is continuous, so that we can apply the Reshetnjak Theorem (see, e.g., [3, Th. 1.3.2]) to obtain

$$
\lim _{k \rightarrow+\infty} F_{0}\left(A_{k}\right)=F_{0}(A) .
$$

Then, the lower-semicontinuity of the upper $\Gamma$-limit allows to conclude the proof.

\section{References}

[1] R. Alicandro, A. Braides, and M. Cicalese. Phase and anti-phase boundaries in binary discrete systems: a variational viewpoint. Netw. Heterog. Media 1 (2006), 85-107.

[2] A.S. Besicovitch. Almost Periodic Functions, Dover, Cambridge, 1954.

[3] A. Braides, Approximation of Free-Discontinuity Problems, Lecture Notes in Mathematics 1694, Springer Verlag, Berlin, 1998.

[4] A. Braides. Г-convergence for Beginners, Oxford University Press, Oxford, 2002.

[5] A. Braides. A handbook of $\Gamma$-convergence. In Handbook of Differential Equations. Stationary Partial Differential Equations, Volume 3 (M. Chipot and P. Quittner, eds.), Elsevier, 2006.

[6] A. Braides and A. Defranceschi. Homogenization of Multiple Integrals, Oxford University Press, Oxford, 1998.

[7] A. Braides, I Fonseca, and G. Francfort. 3D-2D asymptotic analysis for inhomogeneous thin films. Indiana Univ. Math. J. 49 (2000), 1367-1404.

[8] A. Braides, M. Maslennikov, and L. Sigalotti. Homogenization by blow-up. Applicable Anal. 87 (2008), 1341-1356.

[9] A. Braides and A. Piatnitski. Homogenization of surface and length energies for spin systems, to appear.

[10] A. Braides, G. Riey, and M. Solci. Homogenization of Penrose tilings. C.R. Acad. Sci. Paris, Ser. I 347 (2009), 697-700.

[11] A. Braides and M. Solci. Interfacial energies on Penrose lattices. Math. Mod. Meth. Appl. Sci (2011), in print.

[12] G. Dal Maso. An Introduction to Г-convergence, Birkhauser, Boston, 1993. 
[13] N.G. de Bruijn. Algebraic theory of Penrose's nonperiodic tilings of the plane. Proc. K. Ned. Akad. Wet. Ser. A 43 (1981), 39-66.

[14] I. Fonseca and S. Müller. Quasiconvex integrands and lower semicontinuity in $L^{1}$. SIAM J. Math. Anal. 23 (1992), 1081-1098.

[15] M. Senechal, Quasicrystals and Geometry, Cambridge University Press, Cambridge, 1995. 